\title{
D-dimer plasmatic levels as a marker for diagnosis and prognosis of hepatocellular carcinoma patients with portal vein thrombosis
}

This article was published in the following Dove Press journal:

Clinical and Experimental Gastroenterology

\author{
Michele Malaguarnera ${ }^{1,2}$ \\ Saverio Latteri ${ }^{3}$ \\ Gaetano Bertino ${ }^{4}$ \\ Roberto Madeddu ${ }^{5}$ \\ Vito Emanuele Catania ${ }^{3}$ \\ Giuseppe Currò ${ }^{6}$ \\ Antonio Maria Borzi \\ Filippo Drago' \\ Giulia Malaguarnera' \\ 'Department of Biomedical and \\ Biotechnological Science, University \\ of Catania, Catania, Italy; ${ }^{2}$ Research \\ Center "The Great Senescence", \\ University of Catania, Catania, Italy; \\ ${ }^{3}$ Department of Medical, Surgical \\ Sciences and Advanced Technologies \\ "Gian Filippo Ingrassia", University of \\ Catania, Catania, Italy; ${ }^{4}$ Department of \\ Experimental and Clinical Medicine, \\ University of Catania, Catania, Italy; \\ ${ }^{5}$ Department of Biomedical Sciences, \\ University of Sassari, Sassari, Italy; \\ ${ }^{6}$ Department of Human Pathology, \\ University of Messina, Messina, Italy
}

Correspondence: Giulia Malaguarnera Department of Biomedical and Biotechnological Science, Via Santa Sofia, 78, University of Catania, Catania, 95123, Italy

Email giulia.malaguarnera@live.it
Purpose: Portal vein thrombosis (PVT) is one of the severe complications of hepatocellular carcinoma (HCC). PVT deteriorates the liver, and its dysfunction increases the risk of bleeding, influencing the prognosis of patients with liver cirrhosis and HCC. The aim of our study was to investigate whether D-dimer testing could be a sensitive marker for the diagnosis and prognosis of HCC patients with PVT.

Patients and methods: Between June 2010 and December 2015, 118 HCC patients were admitted to Cannizzaro Hospital, Catania, and 50 controls were recruited from their relatives for health examinations. All enrolled patients were diagnosed and pathologically confirmed as having HCC. D-dimer was measured with an enzyme-linked immunosorbent assay using 2 monoclonal antibodies against nonoverlapping determinants of D-dimer.

Results: D-dimer levels in HCC patients with PVT were significantly higher vs HCC patients without PVT, $P<0.002$, and vs controls, $P<0.001$.

Conclusion: Plasma D-dimer is a sensitive marker of fibrin turnover and allows for the recognition of activated coagulation which may be manifested in HCC with PVT.

Keywords: hepatocellular carcinoma, D-dimer, portal venous thrombosis, coagulation, cancer, venous thromboembolism

\section{Introduction}

Hepatocellular carcinoma (HCC) represents a challenging malignancy of worldwide importance and is the second most common cause of cancer-related death globally and the ninth leading cause of cancer death in the USA. ${ }^{1}$ The number of deaths per year attributed to the liver cancer is almost identical to its incidence. ${ }^{2}$ Incidence of HCC is rapidly increasing in the United States, and it is projected to become the third leading cause of cancer-related death by 2030 . The prognosis depends on tumor stage at diagnosis, with curative options only available for patients diagnosed at an early stage.,4

Portal vein thrombosis (PVT) is one of the severe complications of HCC. The prevalence of PVT is $>0.5 \%$ in patients with hepatic cirrhosis and in patients with HCC. ${ }^{5-7}$

PVT deteriorates the liver dysfunction, increases the risk of bleeding, and influences the prognosis of patients with liver cirrhosis and HCC. ${ }^{8}$

Many potential prothrombotic factors exist, such as lipoprotein(a), homocystein, folate deficiency, and dyslipidemia; ${ }^{9-13}$ one such plasma factor is the fibrin D-dimer fragment. ${ }^{14,15}$ Activation of coagulation and fibrinolysis are often associated with malignancies and involved in angiogenesis, tumor cell invasion, tumor progression, and prognosis. ${ }^{16}$ 
D-dimer is a stable end product of fibrin degradation and is converted from fibrinogen by activated thrombin, and so D-dimer levels rise with fibrinolysis. ${ }^{17-19}$

D-dimer reflects intravascular turnover of fibrin by identifying the presence of cross-linked fibrin degradation products without interference from fibrinogen or non-crosslinked fibrin. ${ }^{20,21}$ Cross-linked fibrin in the extracellular matrix serves as a stable framework for endothelial cell migration during angiogenesis and tumor cell migration during invasion.

D-dimer as marker of endogenous fibrinolysis should be detectable in patients with PVT and can predict disease progression and prognosis. ${ }^{22}$

We hypothesized that the use of D-dimer testing in patients with suspected PVT would reduce the need of ultrasound imaging and rule out PVT in a higher proportion of patients on the day of presentation.

We investigated also the prognostic factors and clinicopathological parameters in patients with high levels of D-dimer.

\section{Methods}

\section{Patient recruitment}

We conducted an observational study in the period between June 2010 and December 2015. We observed 118 HCC patients (58 with PVT and 60 without PVT) who were admitted to Cannizzaro Hospital, Catania, and 50 controls were recruited from their relatives for health examination.

All enrolled patients were diagnosed and pathologically confirmed as HCC.

The diagnosis of HCC was confirmed either pathologically or by using images obtained from computer tomography or magnetic resonance imaging. The diagnosis was based on the typical hallmark of HCC, which is the presence of hypervascularity in the arterial phase with a washout in the portal venous or delayed phases. ${ }^{23}$

Using these imaging techniques, we evaluated also tumorrelated variables, including the maximum tumor diameter, the number of tumors, and the presence of extrahepatic metastases. The clinical stage was determined according to the TNM Classification system. ${ }^{24}$

Liver cirrhosis was diagnosed with histological, clinical, biochemical, and morphological criteria.

This study complied with the standards of the declaration of Helsinki as well as current ethical guidelines and was approved by the institutional ethical board of Cannizzaro Hospital. Written informed consent for participation in the study was obtained from HCC patients and control subjects.
Patients with clinical and/or histologically proven $\mathrm{HCC}$ with or without cirrhosis were included in this study.

Patients with the following conditions were excluded from the study:

1. significant metabolic, renal, or gastrointestinal disease;

2. coagulation problem;

3. history of acute peptic ulcer disease;

4. collagen disease, chronic infections.

Demographic data, etiology, and duration from first scan to diagnosis of HCC were collected. TNM stage, $\alpha$-fetoprotein level, Model for End-stage Liver Disease score, Child-Pugh score, and time to diagnosis were determined.

The presence or absence of cirrhosis was identified. The Cancer of the Liver Italian Program (CLIP) score was also evaluated. ${ }^{25}$ CLIP score is used for the prognosis in patients with HCC and it is calculated by assigning a score $(0,1$, or 2) to each of 4 clinical factors: 1) Child-Pugh stage, 2) number of tumors nodules and whether tumors extend, 3) $\alpha$-fetoprotein, and 4) PVT.

Control subjects were healthy subjects without any history of cancer, major organ failure (eg, heart, brain, lung, kidney, or liver), or active intravenous drug abuse.

\section{Blood collection and processing}

Blood samples were obtained after the patients had fasted for 12 hours overnight. Venous blood samples were taken from all patients between 8 am and $10 \mathrm{am}$.

After informed consent, blood was drawn without stasis by clean venepuncture and collected into vacutainer tubes containing $1 / 10$ volume of $0.105 \mathrm{~mol} / \mathrm{L}$ trisodium citrate.

Full blood count, platelet count, prothrombin time (PTT) or international normalized ratio, and PTT were measured using an automatic coagulometer.

Albumin, aspartate aminotransferase, alanine aminotransferase, $\gamma$-glutamil transpeptidase, and $\alpha$-fetoprotein were assessed on admission to the hospital.

D-dimer was measured with an enzyme-linked immunosorbent assay (ELISA) using 2 monoclonal antibodies against nonoverlapping determinants of D-dimer.

The analytic coefficient of variation was from $6.5 \%$ to $9.4 \%$, from higher to lower D-dimer concentration.

\section{Doppler measurements}

All subjects were examined after overnight fasting in a supine position after a rest of 15 minutes, in order to avoid any influence of food, posture, and exercise. The portal vein was examined following guidelines. ${ }^{26}$ 
Care was taken to ensure that the angle of insonation between the Doppler beam and the portal trunk was between $30^{\circ}$ and $60^{\circ}$. The Doppler study was performed by positioning the sample volume at about the middle of the portal trunk (between the liver hilum and the splenomesenteric junction) by approaching from the epigastrium with the probe placed slightly obliquely.

The instrument using time-averaged maximum velocity calculated portal flow velocity automatically, which is the parameter providing the most reliable and repeatable results.

\section{Selection and screening of the HCC patients with and without PVT and randomly selected controls}

We studied 58 PVT patients ( 24 females and 30 males) aged 65-78 years (mean age 66.8 \pm 6.71 years), living in Sicily (Italy) and recruited via the Registry Office.

The study groups were as follows:

Group A, 50 randomly selected healthy subjects aged 65-78 years ( 25 female/ 25 males), mean age $68.8 \pm 7.2$ years, composed of blood donors, randomly selected volunteers working at the University of Catania, or their relatives.

Group B were 60 HCC patients (25 females and 35 males), aged $65-76$ years, with a mean age $68.1 \pm 6.10$ years.

\section{Statistical analysis}

We analyzed the data obtained and calculated sensitivity, specificity, positive predictive value, negative predictive value, positive likelihood ratio, and negative likelihood ratio.

The receiver operating characteristic analysis curve was obtained by calculating the sensitivity and specificity of a test at every possible cut-off point and plotting sensitivity against 1-specificity. The curve was used to select optimal cut-off value for a test result.

The diagnostic accuracy was measured by the area under the curve $=50 \%$.

The results are presented as mean $\pm \mathrm{SD}$. The following 2-tailed tests at the $P \leq 0.05$ level of significance were used to evaluate the study: the Mann-Whitney $U$-test was used in case of 2 independent samples and the Spearman's rank correlation coefficient test was used to test for univariate relationship between variables.

Odds ratio and $95 \%$ CI was calculated to study the associations between risk of D-dimer in HCC patients with PVT and in HCC patients without PVT.

Data were analyzed using the statistical package SPSS 7.5 for Windows (SPSS Inc., Chicago, IL, USA).

\section{Results}

The baseline patient characteristics are shown in Table 1. The median age of the patients with PVT was 70 years (range 65-78), the median age of HCC patients without PVT was 71 years (range 65-76), and the median age of control subjects was 70 years (range $65-78$ ). In this study, 69 patients (58.4\%) were males and $49(41.5 \%)$ were females.

Table I Baseline characteristics of the patients

\begin{tabular}{|c|c|c|c|c|c|c|}
\hline \multirow{2}{*}{$\begin{array}{l}\text { Parameter } \\
\text { Female/male [F/M], n }\end{array}$} & \multicolumn{2}{|c|}{$\begin{array}{l}\text { HCC with PVT } \\
(n=58)\end{array}$} & \multicolumn{2}{|c|}{$\begin{array}{l}\text { HCC without PVT } \\
(n=60)\end{array}$} & \multicolumn{2}{|c|}{$\begin{array}{l}\text { Controls } \\
(n=50)\end{array}$} \\
\hline & & $24 / 34$ & & $25 / 35$ & & $25 / 25$ \\
\hline Age (years) [median range] & & $70(65-78)$ & & 71 (65-76) & & $70(65-78)$ \\
\hline Heart rate $(\mathrm{bpm})[$ mean $\pm \mathrm{SD}]$ & & $82.8 \pm 8.10$ & & $84.2 \pm 8.7$ & & $82.1 \pm 7.8$ \\
\hline Systolic blood pressure $(\mathrm{mmHg})[$ mean $\pm \mathrm{SD}]$ & & $136.4 \pm 9.6$ & & $143.4 \pm 8.4$ & & $|4| .2 \pm 9.1$ \\
\hline Diastolic blood pressure $(\mathrm{mmHg})[$ mean $\pm \mathrm{SD}]$ & & $78.6 \pm 8.4$ & & $80.2 \pm 7.9$ & & $78.6 \pm 7.8$ \\
\hline Body mass index $\left(\mathrm{kg} / \mathrm{m}^{2}\right)$ [mean $\left.\pm \mathrm{SD}\right]$ & & $24.7 \pm 4.8$ & & $24.8 \pm 4.7$ & & $24.5 \pm 5.2$ \\
\hline Current/former smokers [n \%] & 28 & 48.27 & 34 & 56.67 & 32 & 64.00 \\
\hline Diabetes mellitus [n \%] & 10 & 17.24 & 10 & 16.67 & 8 & 16.00 \\
\hline Hypertension [n \%] & 15 & 25.86 & 18 & 30.00 & 12 & 24.00 \\
\hline Renal failure [n \%] & 12 & 20.69 & 15 & 25.00 & 8 & 16.00 \\
\hline History of coronary disease [n \%] & 18 & 31.03 & 16 & 26.67 & 6 & 12.00 \\
\hline Heart insufficiency [n \%] & 6 & 10.34 & 12 & 20.00 & 2 & 4.00 \\
\hline Dyspepsia [n \%] & 25 & 43.10 & 14 & 23.33 & 15 & 30.00 \\
\hline Alcohol consumption [n \%] & 24 & 41.38 & 19 & 31.67 & 10 & 20.00 \\
\hline Hepatitis C virus (HCV) [n \%] & 20 & 34.48 & 21 & 35.00 & - & - \\
\hline Hepatitis B virus (HBV) [n \%] & 18 & 31.03 & 20 & 33.33 & - & - \\
\hline Unknown etiology [n \%] & 16 & 27.59 & 19 & 31.67 & - & - \\
\hline
\end{tabular}

Abbreviations: HCC, hepatocellular carcinoma; PVT, portal vein thrombosis. 
Antibodies to hepatitis $\mathrm{C}$ virus were found in 41 patients (34.7\%), and 38 patients $(32.2 \%)$ were positive for the hepatitis B surface antigen. The remaining 39 patients (33\%) were negative for both antibodies to hepatitis $\mathrm{C}$ virus and hepatitis B surface antigen. The diagnosis of HCC was confirmed histologically in 49 patients (41.5\%), whereas the diagnosis was made using imaging techniques in the remaining patients (Table 1).

A preserved liver function, indicated by a Child-Pugh grade $\mathrm{A}$, was present in 45 patients $(38.1 \%)$, while 62 patients $(52.5 \%)$ were classified as Stage I or II.

In our study, D-dimer presented a positive predictive value 0.6 (95\% CI $0.53-0.66)$, negative predictive value 0.34 (95\% CI $0.24-0.47$ ), sensitivity 0.62 (95\% CI $0.49-0.73$ ), specificity 0.33 (95\% CI 0.20-0.49), and accuracy 0.51 (95\% CI 0.41-0.60).

\section{Correlation between HCC patients with PVT, HCC patients without PVT, and controls}

The following correlations were observed:

1) The comparison between HCC patients with PVT and HCC patients without PVT showed that D-dimer was higher, ie, $384 \mathrm{ng} / \mathrm{mL} P<0.002$, bilirubin was $0.40 \mathrm{mg} /$ $\mathrm{dL} P<0.001$; international normalized ratio $0.64 P<0.001$, and $\alpha$-fetoprotein $129 \mathrm{mg} / \mathrm{L} P<0.001$; albumin was found to be the lowest, ie, $0.45 \mathrm{~g} / \mathrm{dL} P<0.001$; (Table 2).

2) The comparison between HCC with PVT and controls showed the mean value of $\mathrm{D}$-dimer was higher, being $1.100 \mathrm{ng} / \mathrm{mL} P<0.001$, bilirubin was $1.57 \mathrm{mg} / \mathrm{dL}$ $P<0.001$, international normalized ratio $1.45 P<0.0001$ (95\% CI: 1.27-1.62), and $\alpha$-fetoprotein $324 \mathrm{mg} / \mathrm{L}$ $P<0.001$; albumin was found to be the lowest, ie, $0.91 \mathrm{~g} /$ dL $P<0.001$; (Table 2).
3) The comparison between HCC without PVT vs controls showed a higher D-dimer, of $720 \mathrm{ng} / \mathrm{mL} P<0.001$, bilirubin $1.57 \mathrm{mg} / \mathrm{dL} P<0.001$, international normalized ratio $1.45 P<0.001$, and $\alpha$-fetoprotein $1.95 \mathrm{mg} / \mathrm{L} P<0.001$; albumin was found to be the lowest $0.32 \mathrm{~g} / \mathrm{dL} P<0.004$ (Table 2).

\section{Comparison between HCC patients with high D-dimer levels to normal D-dimer serum levels}

According to the cut-off level, we divided patients in 2 groups:

- $\quad<300 \mathrm{ng} / \mathrm{mL}: \mathrm{n}=43$

- $\geq 300 \mathrm{ng} / \mathrm{mL}: \mathrm{n}=75$

In the comparison of the HCC patients with high D-dimer serum levels vs the HCC patients with normal D-dimer levels, we observed significant levels in PVT patients $(P=0.009)$ with cirrhosis $(P<0.001)$, maximum tumor diameter $(P<0.001)$, tumor invasion $(P=0.02)$, vascular invasion $(P<0.001)$, extrahepatic metastases in Stages III and IV vs Stages I and II $(P<0.01)$, and CLIP 3, 4, 5, and 6 vs CLIP 0, 1, and $2(P<0.001)$.

Elevated D-dimer levels were associated with male sex, advanced age, the presence of cirrhosis, vascular invasion, and the presence of extrahepatic metastases. The higher plasma levels of D-dimer were associated with higher ChildPugh grades and higher CLIP score.

Impaired liver function was reported to accompany higher dimer serum levels. In the present study, the dimer levels were associated with changes in levels of albumin, hemoglobin, aspartate aminotransferase, $\gamma$-glutamil transpeptidase, and $\alpha$-fetoprotein. Our data show that serum dimer increased as HCC and CLIP score increased.

Table 2 D-dimer and laboratory parameter

\begin{tabular}{llll}
\hline Parameter & $\begin{array}{l}\text { HCC with PVT } \\
(\mathbf{n = 5 8 )}\end{array}$ & $\begin{array}{l}\text { HCC without PVT } \\
(\mathbf{n = 6 0 )}\end{array}$ & Controls (n=50) \\
\hline D-dimer $\mathrm{ng} / \mathrm{mL}$ & $1.344 \pm 64$ & $960 \pm 71$ & $240 \pm 22$ \\
Bilirubin $\mathrm{mg} / \mathrm{dL}$ & $2.66 \pm 0.56$ & $2.25 \pm 0.70$ & $1.08 \pm 0.44$ \\
Albumin g/dL & $3.14 \pm 0.68$ & $3.56 \pm 0.48$ & $3.88 \pm 0.67$ \\
$\alpha$-Fetoprotein mg/L & $287.4 \pm 16.24$ & $277.6 \pm 17.88$ & $3.80 \pm 0.70$ \\
Aspartate aminotransferase IU/L & $58 \pm 10$ & $55 \pm 12$ & $36 \pm 8$ \\
Alanine aminotransferase IU/L & $5 I \pm I 1$ & $50 \pm 12$ & $33 \pm 7$ \\
$\gamma$-Glutamil transpeptidase IU/L & $67 \pm 12$ & $65 \pm 11$ & $31 \pm 9$ \\
INR & $2.70 \pm 0.50$ & $2.12 \pm 0.61$ & $1.30 \pm 0.29$ \\
\hline
\end{tabular}

Note: Data shown as mean \pm SD.

Abbreviations: HCC, hepatocellular carcinoma; INR, international normalized ratio; PVT, portal vein thrombosis. 


\section{Discussion}

D-dimer, a fibrin degradation product, is elevated by increasing fibrin formation and fibrinolysis. ${ }^{27}$

Coagulation and fibrinolysis markers have the potential to serve as predictors of disease and disease severity.

The pathogenesis of PVT in patients with liver cirrhosis and with HCC remains unclear, although the sluggish reduction of portal vein blood flow and the presence of periportal lymphangitis and fibrosis may be important. ${ }^{28-32}$

In an autopsy series of 254 patients with PVT, 72 (28\%) of the PVT patients had cirrhosis, and one third of those also had primary hepatic cancer. In particular, all 59 PVT patients (23\%) had primary hepatobiliary cancer and 111 (44\%) had secondary malignancy of the hepatobiliary region. ${ }^{34}$

Activation of coagulation and fibrinolysis is known to be associated with cancer. This finding raises the possibility that high dimer could be an intrinsic consequence of tumor growth. Although the mechanisms that promote human HCC malignancy remain unclear, cancer metastases is thought to involve a complex multistep pathway including growth, angiogenesis, dissemination, invasion, and survival of cancer cells ${ }^{35}$ (Table 3 ).
Levels of plasma fibrin D-dimer have been studied in patients with various malignancies, where they have suggested being an indicator of tumor progression, remission, or treatment response. Patients with increased D-dimer concentrations may have hyper-fibrinolysis, including primary coagulation activation-delayed hepatic clearance of tissue plasminogen activator and reduced hepatic synthesis of fibrinolytic inhibitor. D-dimer levels can be utilized as a predictor marker for outcomes of HCC, to explore more simple and accurate prognostic factors from the clinical routine examinations.

Prothrombotic tendency in tumors is suggested to be related to the ability of tumor cells to produce and secrete procoagulant/fibrinolytic substances and inflammatory cytokines as well as the physical interaction between tumor and vascular cells. ${ }^{36}$ The extent of such activation has been reported to correlate with tumor stage and prognosis in HCC. ${ }^{37}$ D-dimer assays have been explored as a tool for a diagnosis of thromboembolism. D-dimer fragment is produced during the degradation of thrombin-generated fibrin clots by plasmin.

Table 3 Clinical features of the HCC patients: D-dimer and laboratory parameters

\begin{tabular}{|c|c|c|c|c|c|}
\hline \multirow[t]{2}{*}{ Variables } & \multicolumn{2}{|c|}{ D-dimer $<300$} & \multicolumn{2}{|c|}{ D-dimer $\geq 300$} & \multirow[t]{2}{*}{$P$-value } \\
\hline & $\mathrm{ng} / \mathrm{mL}$ & $\%$ & $\mathrm{ng} / \mathrm{mL}$ & $\%$ & \\
\hline$\overline{\mathrm{PVT}}$ & & & & & 0.009 \\
\hline Yes & 15 & 34.88 & 45 & 60.00 & \\
\hline No & 28 & 65.12 & 30 & 40.00 & \\
\hline $\mathrm{HCC}$ & & & & & NS \\
\hline I & 14 & 32.56 & 17 & 22.67 & \\
\hline$\|$ & 14 & 32.56 & 15 & 20.00 & \\
\hline III & 10 & 23.26 & 23 & 30.67 & \\
\hline IV & 5 & 11.63 & 20 & 26.67 & \\
\hline CLIP score & & & & & $<0.001$ \\
\hline $0-2$ & 25 & 58.14 & 14 & 18.67 & \\
\hline $3-6$ & 18 & 41.86 & 61 & 81.33 & \\
\hline Cirrhosis & & & & & $<0.001$ \\
\hline Absent & 28 & 65.12 & 10 & 13.33 & \\
\hline Present & 15 & 34.88 & 65 & 86.67 & \\
\hline Maximum tumor diameter & & & & & $<0.001$ \\
\hline$<50 \mathrm{~mm}$ & 33 & 76.74 & 30 & 40.00 & \\
\hline$\geq 50 \mathrm{~mm}$ & 10 & 23.26 & 45 & 60.00 & \\
\hline Tumor invasion & & & & & $<0.05$ \\
\hline Absent & 27 & 62.79 & 30 & 40.00 & \\
\hline Present & 16 & 37.21 & 45 & 57.33 & \\
\hline Vascular invasion & & & & & $<0.001$ \\
\hline Absent & 25 & 58.14 & 20 & 26.67 & \\
\hline Present & 18 & 41.86 & 55 & 73.33 & \\
\hline Extrahepatic metastasis & & & & & $<0.001$ \\
\hline Absent & 30 & 69.77 & 23 & 30.67 & \\
\hline Present & 13 & 30.23 & 52 & 69.33 & \\
\hline
\end{tabular}

Note: Values in bold are $P<0.01$.

Abbreviations: CLIP, Cancer of the Liver Italian Program; HCC, hepatocellular carcinoma; NS, not significant; PVT, portal vein thrombosis. 
An increasing body of evidence suggests the existence of a relationship between activation of coagulation and tumor angiogenesis, progression, and metastatic spread. ${ }^{38,39}$ Factors that may impair the hemostatic balance in patients with HCC are liver related or bacterial infections induced. ${ }^{40-42}$ Frequent bacterial infections may promote a hemorrhagic diathesis through a direct effect of endotoxin or the hyperproduction of proinflammatory cytokines, causing defect in platelet aggregation, coagulation activation, hyperfibrinolysis, and release of endogenous heparinoids. ${ }^{43}$

In a prospective study, D-dimer levels were high in cancer patients with suspected PVT while radiologic testing excluded the diagnosis of venous thromboembolism. ${ }^{44}$

Duplex ultrasonography remains the principal tool for the diagnosis of PVT; it allows both an analysis of venous compressibility and Doppler imaging of venous blood flow. ${ }^{45,46}$ The portal flow velocity is an important predictive factor for PVT development, especially when portal flow velocity is lower than $15 \mathrm{~cm} / \mathrm{s}$ on Doppler ultrasound evaluation. ${ }^{47}$

The advantages of ultrasonography include its accuracy in the diagnosis of PVT without intravenous contrast agents, its ability to be done at the bedside, and its lower cost. ${ }^{48,49}$

\section{Conclusion}

More recently, thrombogenic and inflammatory risk factors have also been implicated in the pathogenesis of PVT. In selected populations, an association between plasma D-dimer level and an increased risk of PVT thrombosis has been proposed. Plasma D-dimer has an important role as a marker of thrombogenesis. It is a sensitive marker of fibrin turnover and allows the recognition of activated coagulation, which may be manifested in various clinical conditions. ${ }^{50,51}$ The measurement may allow the specific and rapid evaluation of coagulofibrinolytic activation, which suggests a prothrombotic or hypercoagulable state.

Previous studies have reported that high level of plasma D-dimer is associated with poor prognosis in several solid malignancies. ${ }^{52-56}$

The limitation of this study is the relatively small sample size, which reduces the statistical power for subgroup analysis. The results of this study should be evaluated with some caution because all patients were from a single medical center.

D-dimer might reflect the presence of other environmental risk factors for thrombosis, and higher D-dimer concentration may be associated with smoking status and some coagulation and inflammation markers. D-dimer is associated with an increased risk of thrombotic events, irrespective of baseline vascular disease, even after adjusting for confounders such as age, smoking, and diabetes.
Further research is needed on the generalizability of our finding. A longitudinal design and an interventional study with a large sample may help determine the optimal strategies to improve high D-dimer levels in HCC patients.

\section{Disclosure}

The authors report no conflicts of interest in this work.

\section{References}

1. El-Serag HB. Hepatocellular carcinoma. N Engl J Med. 2011;365(12): 1118-1127.

2. Mittal S, El-Serag HB. Epidemiology of hepatocellular carcinoma: consider the population. J Clin Gastroenterol. 2013;47 Suppl(Suppl):S2-6.

3. Rapisarda V, Loreto C, Malaguarnera M, et al. Hepatocellular carcinoma and the risk of occupational exposure. World J Hepatol. 2016;8(13):5731948-19905182.

4. Yopp AC, Mansour JC, Beg MS, et al. Establishment of a multidisciplinary hepatocellular carcinoma clinic is associated with improved clinical outcome. Ann Surg Oncol. 2014;21(4):1287-1295. Epub 2013 Dec 7.

5. Zhang D, Hao J, Yang N, Protein C. Protein C and D-dimer are related to portal vein thrombosis in patients with liver cirrhosis. $J$ Gastroenterol Hepatol. 2010;25(1):116-121. Epub 2009 Aug 3.

6. Northup PG, Mcmahon MM, Ruhl AP, et al. Coagulopathy does not fully protect hospitalized cirrhosis patients from peripheral venous thromboembolism. Am J Gastroenterol. 2006;101(7):1524-1528.

7. Gulley D, Teal E, Suvannasankha A, Chalasani N, Liangpunsakul S. Deep vein thrombosis and pulmonary embolism in cirrhosis patients. Dig Dis Sci. 2008;53(11):3012-3017. Epub 2008 Apr 29.

8. Malaguarnera G, Catania VE, Francaviglia A, et al. Lipoprotein(a) in patients with hepatocellular carcinoma and portal vein thrombosis. Aging Clin Exp Res. 2017;29(Suppl 1):185-190.

9. Galvano F, Malaguarnera M, Vacante M, et al. The physiopathology of lipoprotein (a. Front Biosci. 2010;2(2):866-875.

10. Tripodi A, Anstee QM, Sogaard KK, Primignani M, Valla DC. Hypercoagulability in cirrhosis: causes and consequences. JThromb Haemost. 2011;9(9):1713-1723.

11. Malaguarnera G, Gagliano C, Bucolo C, et al. Lipoprotein(a) Serum Levels in Diabetic Patients with Retinopathy. Bio Med Res Int. 2013;2013:943505.

12. Amitrano L, Guardascione MA, Brancaccio V, et al. Risk factors and clinical presentation of portal vein thrombosis in patients with liver cirrhosis. J Hepatol. 2004;40(5):736-741.

13. Malaguarnera G, Gagliano C, Salomone S, et al. Folate status in type 2 diabetic patients with and without retinopathy. Clin Ophthalmol. 2015;9:1437-1442.

14. Sakkinen PA, Macy EM, Callas PW, et al. Analytical and biologic variability in measures of hemostasis, fibrinolysis, and inflammation: assessment and implications for epidemiology. Am J Epidemiol. 1999;149(3):261-267.

15. Tripodi A, Mannucci PM. The coagulopathy of chronic liver disease. N Engl J Med. 2011;365(2):147-156.

16. Korte W. Changes of the coagulation and fibrinolysis system in malignancy: their possible impact on future diagnostic and therapeutic procedures. Clin Chem Lab Med. 2000;38(8):679-692.

17. Uccello M, Malaguarnera G, Pelligra EM, Biondi A, Basile F, Motta M. Lipoprotein(a) as a potential marker of residual liver function in hepatocellular carcinoma. Indian J Med Paediatr Oncol. 2011;32(2): 71-75.

18. Malaguarnera G, Gagliano C, Giordano M, et al. Homocysteine serum levels in diabetic patients with non proliferative, proliferative and without retinopathy. Biomed Res Int. 20142014;2014:1-4e191497.

19. Tripodi A. D-dimer testing in laboratory practice. Clin Chem. 2011;57(9): $1256-1262$. 
20. Lip GY, Lowe GD. Fibrin D-dimer: a useful clinical marker of thrombogenesis? Clin Sci. 1995;89(3):205-214.

21. Hunt FA, Rylatt DB, Hart RA, Bundesen PG. Serum crosslinked fibrin (XDP) and fibrinogen/fibrin degradation products (FDP) in disorders associated with activation of the coagulation or fibrinolytic systems. Br J Haematol. 1985;60(4):715-722.

22. Zacharoulis D, Hatzitheofilou C, Athanasiou E, Zacharoulis S. Antiangiogenic strategies in hepatocellular carcinoma: current status. Expert Rev Anticancer Ther. 2005;5(4):645-656.

23. European Association For The Study OfThe Liver, European Organisation For Research And Treatment Of Cancer. EASL-EORTC clinical practice guidelines: management of hepatocellular carcinoma. $J$ Hepatol. 2012;56(4):908-943.

24. Minagawa M, Ikai I, Matsuyama Y, Yamaoka Y, Makuuchi M. Staging of hepatocellular carcinoma: assessment of the Japanese TNM and AJCC/ UICC TNM systems in a cohort of 13,772 patients in Japan. Ann Surg. 2007;245(6):909-922.

25. [No authors listed]. A new prognostic system for hepatocellular carcinoma: a retrospective study of 435 patients: the Cancer of the Liver Italian Program (CLIP) investigators. Hepatology. 1998;28(3):751-755.

26. Sabbà $\mathrm{C}$, Merkel $\mathrm{C}$, Zoli $\mathrm{M}$, et al. Interobserver and interequipment variability of echo-Doppler examination of the portal vein: effect of a cooperative training program. Hepatology. 1995;21(2):428-433.

27. Stender MT, Larsen AC, Sall M, Thorlacius-Ussing O. D-Dimer predicts prognosis and non-resectability in patients with pancreatic cancer: a prospective cohort study. Blood Coagul Fibrinolysis. 2016;27(5):597-601.

28. Malaguarnera G, Vacante M, Drago F, et al. Endozepine-4 levels are increased in hepatic coma. World J Gastroenterol. 2015;21(30): 9103-9110.

29. Connolly GC, Chen R, Hyrien O, et al. Incidence, risk factors and consequences of portal vein and systemic thromboses in hepatocellular carcinoma. Thromb Res. 2008;122(3):299-306.

30. Delahousse B, Labat-Debelleix V, Decalonne L, D'Alteroche L, Perarnau JM, Gruel Y. Comparative study of coagulation and thrombin generation in the portal and jugular plasma of patients with cirrhosis. Thromb Haemost. 2010;104(4):741-749.

31. Palareti G, Legnani C, Mazziotti A, Zanello M, Sama C, Coccheri S. The "lytic state" during orthotopic liver transplantation: plasminogen activators and other protease activities. Semin Thromb Hemost. 1993;19(3):290-291.

32. Malaguarnera M, Vacante M, Russo C, et al. Rosuvastatin reduces nonalcoholic fatty liver disease in patients with chronic hepatitis $\mathrm{C}$ treated with $\alpha$-interferon and ribavirin: Rosuvastatin reduces NAFLD in HCV patients. Hepat Mon. 2011;11(2):92-98.

33. Ogren M, Bergqvist D, Björck M, Acosta S, Eriksson H, Sternby NH. Portal vein thrombosis: prevalence, patient characteristics and lifetime risk: a population study based on 23,796 consecutive autopsies. World J Gastroenterol. 2006;12(13):2115-2119.

34. Pirisi M, Avellini C, Fabris C, et al. Portal vein thrombosis in hepatocellular carcinoma: age and sex distribution in an autopsy study. J Cancer Res Clin Oncol. 1998;124(7):397-400.

35. Budhu AS, Zipser B, Forgues M, Ye QH, Sun Z, Wang XW. The molecular signature of metastases of human hepatocellular carcinoma. Oncology. 2005;69(Suppl 1):23-27.

36. Zhang B, Liu G, Liu X, Zheng S, Dong K, Dong R. Circulating D-dimer level correlates with disease characteristics in hepatoblastoma patients. Medicine. 2017;96(47):e8798.

37. Sohne M, Kruip MJ, Nijkeuter M, et al. Accuracy of clinical decision rule, D-dimer and spiral computed tomography in patients with malignancy, previous venous thromboembolism, COPD or heart failure and in older patients with suspected pulmonary embolism. J Thromb Haemost. 2006;4(5):1042-1046.
38. Man YN, Wang YN, Hao J, et al. Pretreatment plasma D-dimer, fibrinogen, and platelet levels significantly impact prognosis in patients with epithelial ovarian cancer independently of venous thromboembolism. Int J Gynecol Cancer. 2015;25(1):24-32.

39. Lima LG, Monteiro RQ. Activation of blood coagulation in cancer: implications for tumour progression. Biosci Rep. 2013;33(5): 701-710.

40. Zhang X, Long Q. Elevated serum plasma fibrinogen is associated with advanced tumor stage and poor survival in hepatocellular carcinoma patients. Medicine. 2017;96(17):e6694.

41. Malaguarnera G, Pennisi M, Bertino G, et al. Resveratrol in Patients with Minimal Hepatic Encephalopathy. Nutrients. 2018;10(3):329.

42. di Rosa M, Malaguarnera G, de Gregorio C, D’Amico F, Mazzarino MC, Malaguarnera L. Modulation of chitotriosidase during macrophage differentiation. Cell Biochem Biophys. 2013;66(2):239-247.

43. Malaguarnera M, Vacante M, Condorelli G, et al. Probiotics and prebiotics in the management of constipation in the elderly. Acta Medica Mediterranea. 2013;791.

44. Knowlson L, Bacchu S, Paneesha S, Mcmanus A, Randall K, Rose P. Elevated D-dimers are also a marker of underlying malignancy and increased mortality in the absence of venous thromboembolism. J Clin Pathol. 2010;63(9):818-822.

45. Zierler BK. Ultrasonography and diagnosis of venous thromboembolism. Circulation. 2004;109(12 Suppl 1):I-9--0.

46. Zocco MA, di Stasio E, de Cristofaro R, et al. Thrombotic risk factors in patients with liver cirrhosis: correlation with MELD scoring system and portal vein thrombosis development. J Hepatol. 2009;51(4): 682-689.

47. Khalil J, Bensaid B, Elkacemi H, et al. Venous thromboembolism in cancer patients: an underestimated major health problem. World $J$ Surg Oncol. 2015;13:204.

48. Latteri S, Malaguarnera G, Mannino M, et al. Ultrasound as point of care in management of polytrauma and its complication. J Ultrasound. 2017;20(2):171-177.

49. Gaitini D, Beck-Razi N, Haim N, Brenner B. Prevalence of upper extremity deep venous thrombosis diagnosed by color Doppler duplex sonography in cancer patients with central venous catheters. $J$ Ultrasound Med. 2006;25(10):1297-1303.

50. Kanne JP, Lalani TA. Role of computed tomography and magnetic resonance imaging for deep venous thrombosis and pulmonary embolism. Circulation. 2004;109(12 Suppl 1):I15-21.

51. Cooper DL, Sandler AB, Wilson LD, Duffy TP. Disseminated intravascular coagulation and excessive fibrinolysis in a patient with metastatic prostate cancer. Response to epsilon-aminocaproic acid. Cancer. 1992;70(3):656-658.

52. Fukumoto K, Taniguchi T, Usami N, et al. Preoperative plasma D-dimer level is an independent prognostic factor in patients with completely resected non-small cell lung cancer. Surg Today. 2015;45(1):63-67.

53. Zhang PP, Sun JW, Wang XY, Liu XM, Li K. Preoperative plasma $\mathrm{D}$-dimer levels predict survival in patients with operable non-small cell lung cancer independently of venous thromboembolism. Eur J Surg Oncol. 2013;39(9):951-956. Epub 2013 Jun 28.

54. Go SI, Lee MJ, Lee WS, et al. D-Dimer Can Serve as a Prognostic and Predictive Biomarker for Metastatic Gastric Cancer Treated by Chemotherapy. Medicine. 2015;94(30):e951.

55. Liu P, Zhang H, Liang X, et al. HBV preS2 promotes the expression of TAZ via miRNA-338-3p to enhance the tumorigenesis of hepatocellular carcinoma. Oncotarget. 2015;6(30):29048-29059.

56. Latteri S, Catania VE, Malaguarnera G, et al. Carcinoembryonic Antigen Serum Levels in Nonmelanoma Skin Cancer. Biomedicines. 2018;6(1):24. 


\section{Publish your work in this journal}

Clinical and Experimental Gastroenterology is an international, peerreviewed, open access, online journal publishing original research, reports, editorials, reviews and commentaries on all aspects of gastroenterology in the clinic and laboratory. This journal is included on PubMed. The manuscript management system is completely online and includes a very quick and fair peer-review system, which is all easy to use. Visit http://www.dovepress.com/testimonials.php to read real quotes from published authors.

Submit your manuscript here: https://www.dovepress.com/clinical-and-experimental-gastroenterology-journal 